\title{
CORRECTION
}

View Article Online

View Journal I View Issue

Check for updates

Cite this: Mater. Horiz., 2020,

7, 3339

DOI: 10.1039/d0mh90071k

rsc.li/materials-horizons

\section{Correction: Biomimetic anti-freezing polymeric hydrogels: keeping soft-wet materials active in cold environments}

\author{
Yukun Jian, ${ }^{\text {ac }}$ Stephan Handschuh-Wang, ${ }^{\text {b }}$ Jiawei Zhang, ${ }^{\text {ac }}$ Wei Lu, ${ }^{\text {ac }}$ \\ Xuechang Zhou ${ }^{* \mathrm{~b}}$ and Tao Chen ${ }^{* a c}$
}

Correction for 'Biomimetic anti-freezing polymeric hydrogels: keeping soft-wet materials active in cold environments' by Yukun Jian et al., Mater. Horiz., 2020, DOI: 10.1039/d0mh01029d.

The authors regret an error in the affiliations listed in the originally published manuscript. The corrected list of affiliations for this paper is as shown above.

The Royal Society of Chemistry apologises for these errors and any consequent inconvenience to authors and readers.

\footnotetext{
${ }^{a}$ Key Laboratory of Marine Materials and Related Technologies, Zhejiang Key Laboratory of Marine Materials and Protective Technologies, Ningbo Institute of Material Technology and Engineering, Chinese Academy of Sciences, Ningbo, 315201, China. E-mail: zhangiiawei@nimte.ac.cn, tao.chen@nimte.ac.cn

${ }^{b}$ College of Chemistry and Environmental Engineering, Shenzhen University, Shenzhen, 518055, China. E-mail: xczhou@szu.edu.cn

${ }^{c}$ University of Chinese Academy of Sciences, 19A Yuquan Road, Beijing, 100049, China.
} 